\title{
Antagonistic antibody prevents toll-like receptor 2 -driven lethal shock-like syndromes
}

\author{
Guangxun Meng, ${ }^{1}$ Mark Rutz, ${ }^{1}$ Matthias Schiemann, ${ }^{1}$ Jochen Metzger, ${ }^{2}$ Alina Grabiec, ${ }^{1}$ \\ Ralf Schwandner, ${ }^{3}$ Peter B. Luppa, ${ }^{2}$ Frank Ebel, ${ }^{4}$ Dirk H. Busch, ${ }^{1}$ Stefan Bauer, ${ }^{1}$ Hermann Wagner, ${ }^{1}$ \\ and Carsten J. Kirschning' \\ ${ }^{1}$ Institute of Medical Microbiology, Immunology, and Hygiene, and 2Institute of Clinical Chemistry and Pathobiochemistry, Technical University of Munich, \\ Munich, Germany. ${ }^{3}$ Tularik GmbH, Regensburg, Germany. ${ }^{4}$ Max von Pettenkofer Institute, Ludwig Maximilian University, Munich, Germany.
}

\begin{abstract}
Hyperactivation of immune cells by bacterial products through toll-like receptors (TLRs) is thought of as a causative mechanism of septic shock pathology. Infections with Gram-negative or Gram-positive bacteria provide TLR2-specific agonists and are the major cause of severe sepsis. In order to intervene in TLR2-driven toxemia, we raised mAb's against the extracellular domain of TLR2. Surface plasmon resonance analysis showed direct and specific interaction of TLR2 and immunostimulatory lipopeptide, which was blocked by T2.5 in a dose-dependent manner. Application of mAb T2.5 inhibited cell activation in experimental murine models of infection. T2.5 also antagonized TLR2-specific activation of primary human macrophages. TLR2 surface expression by murine macrophages was surprisingly weak, while both intra- and extracellular expression increased upon systemic microbial challenge. Systemic application of T2.5 upon lipopeptide challenge inhibited release of inflammatory mediators such as TNF- $\alpha$ and prevented lethal shock-like syndrome in mice. Twenty milligrams per kilogram of T2.5 was sufficient to protect mice, and administration of $40 \mathrm{mg} / \mathrm{kg}$ of T2.5 was protective even 3 hours after the start of otherwise lethal challenge with Bacillus subtilis. These results indicate that epitope-specific binding of exogenous ligands precedes specific TLR signaling and suggest therapeutic application of a neutralizing anti-TLR2 antibody in acute infection.
\end{abstract}

\section{Introduction}

Host cells recognize specific microbial components through pattern recognition receptors (PRRs) such as toll-like receptors (TLRs) that mediate immune responses $(1,2)$. LPS from the outer membrane of Gram-negative bacteria is a potent agonist for TLR4, whose effects on host organisms of different species have been studied extensively in experimental models of infection and septic shock (3-6). Hyperstimulation of host immune cells by microbial products causes the release of large amounts of inflammatory mediators such as the cytokine TNF- $\alpha$ (7). Its systemic presence at high concentrations is recognized as a major cause of septic shock, characterized by clinical parameters such as abnormal coagulation, profound hypotension, and organ failure (8-10). Also, further inflammatory cytokines such as macrophage inhibitory factor have been shown to directly bias host responsiveness to microbial challenge through modulation of TLR expression (11).

The concept of PRR-dependent induction of hyperinflammation by microbial products has been validated using both genetargeted mice lacking the expression of respective receptors, and

Nonstandard abbreviations used: electrophoretic mobility shift assay (EMSA); extracellular domain (ECD); flow cell (FC); heat inactivated (h.i.); human embryonic kidney 293 [cell line] (HEK293); intraperitoneal(ly) (i.p.); leucine-rich repeat (LRR); lipoteichoic acid (LTA); murine toll-like receptor 2 (mTLR2); murine TLR2ECD-human IgGFC $\gamma$ fusion protein (T2EC); $N$-palmitoyl-S-(1,2-bishexadecyloxy-carbonyl)-ethyl-(R)-cysteinyl-seryl-(lysyl)3-lysine (PHCSK 4$) ; N$-palmitoyl-S-(2,3bis(palmitoyloxy)-(2R,S)-propyl)-(R)-cysteinyl-seryl-(lysyl)3-lysine $\left(\mathrm{P}_{3} \mathrm{CSK}_{4}\right)$; pattern recognition receptor (PRR); peptidoglycan $(\mathrm{PGN})$; surface plasmon resonance (SPR); toll-like receptor (TLR)

Conflict of interest: The authors have declared that no conflict of interest exists.

Citation for this article: J. Clin. Invest. 113:1473-1481 (2004).

doi:10.1172/JCI200420762. receptor-specific inhibition of microbial product-induced host cell activation. For example, application of CD14-specific antibodies inhibited LPS-induced cell activation, protected rabbits against LPS-induced pathology, and is being evaluated in clinical trials $(12,13)$. Blockage of further LPS receptors or extracellular effector proteins such as high-mobility group 1 protein has been shown to be preventive as well (14). Another approach of therapeutic intervention in inflammation has been interference with the functions of proinflammatory cytokines such as TNF- $\alpha$ or IL-1 $\beta$. For instance, competitive inhibition of the binding of a cytokine to its signaling receptors by application of recombinant extracellular domain (ECD) or naturalizing receptor antagonist proteins has been shown to be protective in LPS-induced shocklike syndrome (15). Alternatively, antagonistic antibodies targeting cytokines or ECDs of cytokine receptors have been applied for inhibition of inflammatory immune reactions (16). Therapeutic blockage of cytokines such as TNF- $\alpha$ and IL- 6 is used already for treatment of chronic inflammations $(17,18)$.

Besides Gram-negative bacteria, Gram-positive bacteria lacking LPS play an equally important role in the clinical manifestation of shock (10). Cell wall components from these bacteria, such as peptidoglycan (PGN) and lipoteichoic acid (LTA), are considered major causative agents of Gram-positive shock $(19,20)$. PGN is a main component of Gram-positive and is also present in Gramnegative bacterial cell walls, and it consists of an alternating $\beta(1,4)$-linked $N$-acetylmuramyl and $N$-acetylglucosaminyl glycan cross-linked by small peptides (21). In contrast, the macroamphiphile LTA, a saccharide chain molecule consisting of repetitive oligosaccharides connected by alcohols such as ribitol and carrying acyl chains through which it is anchored to the bacterial cell 
membrane, is specific for Gram-positive bacteria (22). For example, LTA has been described to carry the major stimulatory activity of Bacillus subtilis (23). Further, tripalmitoylated proteins, which have been identified in Gram-negative bacteria initially, are mimicked by the synthetic compound $N$-palmitoyl-S-(2,3-bis(palmitoyloxy)(2R,S)-propyl)-(R)-cysteinyl-seryl-(lysyl)3-lysine $\left(\mathrm{P}_{3} \mathrm{CSK}_{4}\right)(24)$.

The bacteria and bacterial products named above are known to trigger the TLR2 signaling cascade (2). For example, the bacterial species Listeria monocytogenes and Staphylococcus aureus, recognized as clinically more important, are phylogenetically closely related to B. subtilis, and the Gram-positive bacteria of all three species produce TLR2 agonists (25-28). However, recent reports indicate that $L$. monocytogenes and $S$. aureus generate additional molecular patterns that elicit immune responses in a TLR2-independent manner in vivo. Susceptibilities of TLR2 $2^{-1}$ as compared with wild-type mice to respective bacterial challenges differed to a limited degree or did not differ (29-31), implicating further PRRs in their cellular recognition. Of note, triacylated $\mathrm{P}_{3} \mathrm{CSK}_{4}$ has been demonstrated to use TLR2 in combination with TLR1, while a diacylated mycoplasmal protein uses TLR6 in addition to TLR2 or cell activation (32-34). The TLR2ECD, whose N-terminal portion has been implicated in direct PGN recognition (35), contains an array of distinct leucine-rich repeat (LRR) motifs. The LRR-rich domain is followed by an LRR C-terminal, a transmembrane, and an intracellular C-terminal toll-IL-1 receptor typical signaling domain (TIR) (36).

Here, we show by application of surface plasmon resonance (SPR) biosensor technology that the TLR2-specific mAb T2.5 abrogated TLR2ECD binding to $\mathrm{P}_{3} \mathrm{CSK}_{4}$. Consequently, TLR2mediated activation of murine and human cells was inhibited in the presence of T2.5, demonstrating ligand binding to a specific epitope within the TLR2ECD to cause signaling-receptor complex formation. Using two different TLR2-dependent shock models, we demonstrate the protective potential of neutralization of TLR2 function with this antibody in vivo. We propose that antagonism of extracellular TLR2ECD function might provide a therapeutic option for prevention of septic shock.

\section{Results}

Application of murine $m A b$ T2.5 for TLR2 expression analysis in vitro. We have selected an IgG1 $\mathrm{\kappa}$ anti-TLR2 mAb named T2.5, which recognized TLR2. Human embryonic kidney 293 (HEK293) cells stably expressing murine or human TLR2 were stained specifically on their surface by T2.5 (Figure 1, A and B). Furthermore, T2.5 did not bind to primary murine TLR2 $2^{--}$but bound to wild-type macrophages cultured in vitro (Figure 1, C and D). T2.5 immunoprecipitated native murine and human TLR2 from lysates of HEK293 cells overexpressing one or the other of the two receptors (Figure 1E). Most importantly, T2.5 precipitated endogenous TLR2 from lysates of RAW264.7 macrophages (Figure 1E). We further analyzed T2.5 for its capacity to specifically detect TLR2 on the subcellular level. Detection of overexpressed murine and human TLR2 was specific (Figure 2A). Further, endogenous TLR2 was detectable on the surface of primary murine human macrophages, as well as within the cytoplasmic space (Figure 2B).

Inbibitory effects of T2.5 on TLR2-specific cell activation in vitro and in vivo. T2.5 inhibited murine and human TLR2-mediated cell activation by the TLR2-specific stimuli $\mathrm{P}_{3} \mathrm{CSK}_{4}$ or $B$. subtilis applied to HEK293 cells overexpressing TLR2, as well as murine RAW264.7 and primary macrophages. NF- $\mathrm{\kappa B}$ activation and IL-8 release, as well as TNF- $\alpha$ and IL- 6 release, respectively, were analyzed upon cellular challenge (Figure 3, A-D, and data not shown). A second newly generated IgG1 $1 \mathrm{~K}$ anti-TLR2 $\mathrm{mAb}$, conT2, was used as a control. This mAb bound to native murine TLR2 (mTLR2), as T2.5 did, but it did not bind to human TLR2 (data not shown) and failed to inhibit TLR2-dependent cell activation in vitro and ex vivo (Figure 3). Also, no inhibition of IL-1 receptor or TLR4 signaling by T2.5 was evident, which indicates that TLR2-independent signaling pathways in T2.5-treated cells remain intact (Figure 3, A-D). Moreover, TLR2-mediated nuclear translocation of NF- $\mathrm{BB}$ was specifically inhibited by T2.5 in human macrophages (Figure 3E). NF-кB-specific electrophoretic mobility shift assay (EMSA), as well as anti-phospho-p38, anti-phospho-Erk1/2, and antiphospho-Akt immunoblot analysis, revealed T2.5 but not conT2 dose-dependent inhibition of $\mathrm{P}_{3} \mathrm{CSK}_{4}$-induced NF- $\mathrm{KB}-\mathrm{DNA}$ binding and cellular kinase phosphorylation (Figure 3, F and G).

Abrogation of TLR2ECD ligand binding by $T 2.5$ and analysis of $T 2.5$ epitope localization. To investigate whether T2.5 blocked binding of TLR2 to its synthetic agonist $\mathrm{P}_{3} \mathrm{CSK}_{4}$, we established an SPR biosensor-based binding assay. $\mathrm{P}_{3} \mathrm{CSK}_{4}$ was immobilized on a chip surface, and binding of murine TLR2ECD-human IgGFC $\gamma$
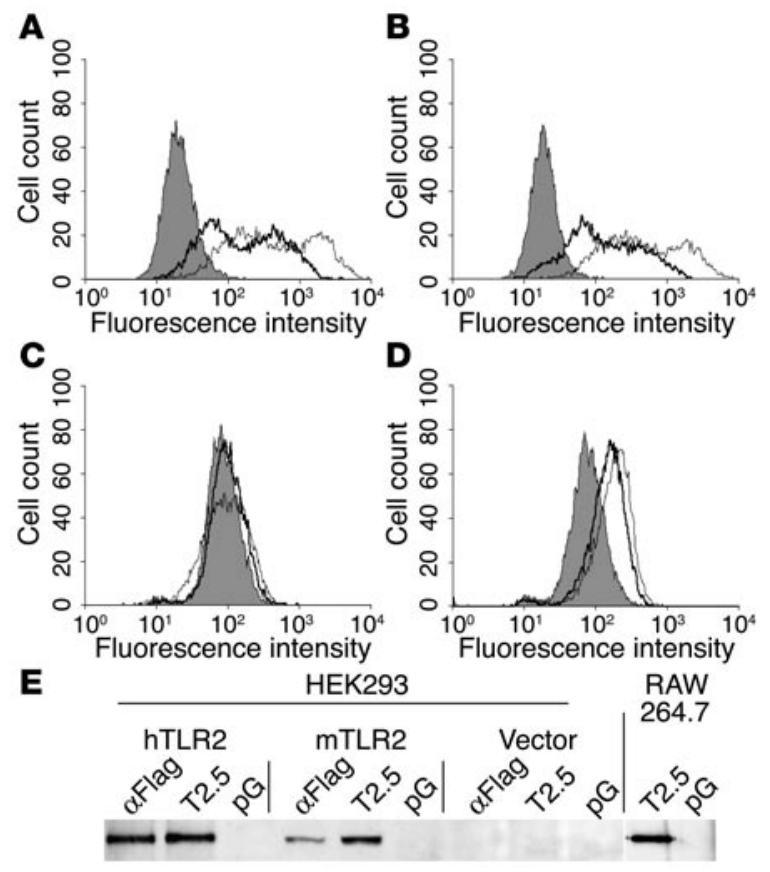

D

Figure 1

Application of mAb T2.5 for specific detection of TLR2. (A-D) Results of flow cytometry of HEK293 cells stably overexpressing Flag-tagged mTLR2 (A) or human TLR2 (B), as well as primary $T L R 2^{-/-}$(C) and wild-type murine macrophages (D), by staining with mAb T2.5 (bold line). Negative controls represent cells incubated with a mouse IgGspecific secondary antibody only (filled areas). For positive controls, Flag-specific (A and B) and mTLR2-specific (C and D) polyclonal antisera were used (thin line). (E) For immunoprecipitation with T2.5, lysates of HEK293 cells overexpressing murine or human TLR2, as well as of murine RAW264.7 macrophages, were applied as indicated. TLR2 precipitates were visualized by application of Flag-specific (HEK293) or mTLR2-specific (RAW264.7) polyclonal antisera. Flagspecific beads ( $\alpha$ Flag) and protein $G$ beads in the absence of antibodies (pG), as well as vector-transfected HEK293 cells, were used as controls. The size of TLR2 was $97 \mathrm{kDa}$. 
A

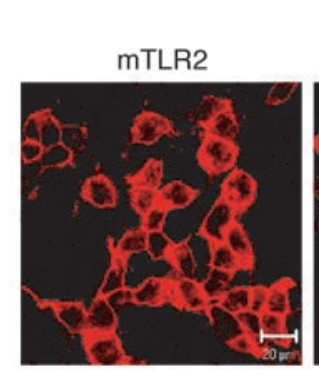

B
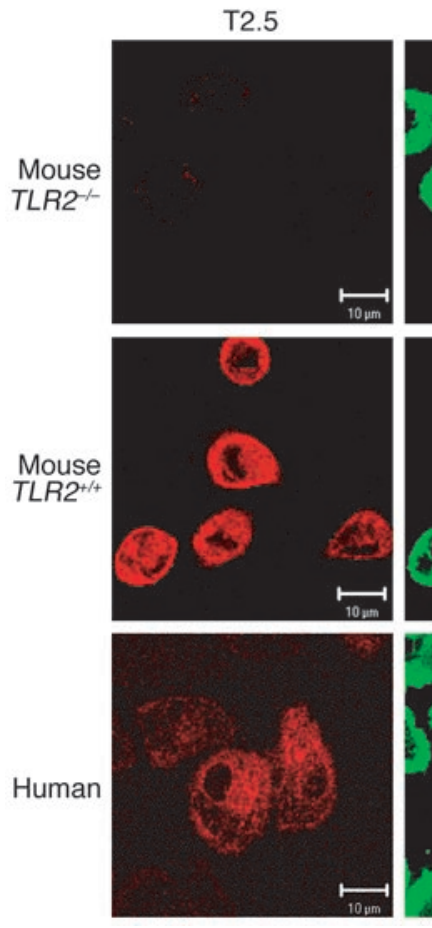

Figure 2

Subcellular localization of TLR2 in vitro. Monoclonal antibody T2.5 was used for cytochemical detection of overexpressed mTLR2 and human TLR2 (hTLR2) (A), as well as endogenous murine ( $T L R 2^{+/+}$, wild-type) or human TLR2 in primary macrophages (B). Vector-transfected HEK293 cells as well as $T L R 2^{-/-}$primary macrophages were analyzed as controls. Concanavalin A (ConA) was used for staining of cellular membranes. The bar in the lower right corner of each field represents a distance of $20 \mu \mathrm{m}$ (A) or $10 \mu \mathrm{m}$ (B) on the slides analyzed.

fusion protein (T2EC) was tested under various conditions. $\mathrm{N}$ palmitoyl-S-(1,2-bishexadecyloxy-carbonyl)-ethyl-(R)-cysteinylseryl-(lysyl)3-lysine $\left(\mathrm{PHCSK}_{4}\right)$, a nonactive analogue of $\mathrm{P}_{3} \mathrm{CSK}_{4}$, was used as a control, and sensorgrams are displayed as subtracted binding curves. Binding of T2EC to $\mathrm{P}_{3} \mathrm{CSK}_{4}$ was specific (Figure $4 \mathrm{~A}$ ). When T2EC was preincubated with T2.5, the antibody dose-dependently inhibited T2EC- ${ }_{3} \mathrm{CSK}_{4}$ binding (Figure 4A). A molar ratio of 3.3 (T2.5/T2EC) was required to reduce binding to $50 \%$. Preincubation of T2EC with T2.5 at tenfold molar excess abrogated T2EC- $\mathrm{P}_{3} \mathrm{CSK}_{4}$ interaction (Figure $4 \mathrm{~A}$ ). In contrast, an isotype-matched control antibody did not block binding of T2EC to $\mathrm{P}_{3} \mathrm{CSK}_{4}$ even when applied at tenfold molar excess (Figure 4B). When applied alone, both $\mathrm{mAb}$ 's did not interact with the sensorchip surface (Figure 4, A and B). The N-terminal third of the LRRrich domain of human TLR2 is not involved in lipopeptide recog- nition (37), and T2.5 cross-reacts with human TLR2 (Figures 2B and 3E). Thus, we applied T2.5 to HEK293 cells overexpressing a mutant construct of human TLR2 that lacks the respective portion of the wild-type ECD (37). Specific abrogation of NF-кB-dependent reporter gene activation upon $\mathrm{P}_{3} \mathrm{CSK}_{4}$ challenge after administration of T2.5 strongly suggests localization of the epitope recognized by T2.5 within the C-terminal portion of the TLR2ECD (Figure 4C).

Surface and intracellular TLR2 expression ex vivo as analyzed immediately after primary cell preparation. Since LPS induces TLR2 expression in primary macrophages in vitro, we first compared T2.5-specific staining of CD $11 b^{+}$splenocytes from LPS-challenged wild-type and $T L R 2^{-/-}$mice by flow cytometry. Weak surface staining and more pronounced intracellular staining were evident (Figure 5A). In subsequent experiments, peritoneal cells and splenocytes from mice infected with Gram-positive B. subtilis bacteria were analyzed. While surface expression of TLR2 in primary murine macrophages was relatively strong upon in vitro culture (Figure 1D), surface expression was weak or not detectable in unchallenged CD $11 b^{+}, \mathrm{CD} 11 \mathrm{c}^{+}, \mathrm{CD} 19^{+}$, and peripheral neutrophil marker $\mathrm{GR}^{+}$subpopulations of splenocytes and peritoneal washout cells (Figure 5, $A$ and $B$, and data not shown). Upon microbial challenge, however, TLR2 ${ }^{+}$cell numbers and TLR2 surface expression increased in $\mathrm{CD} 11 \mathrm{~b}^{+}$and GR $1^{+}$cells (Figure $5 \mathrm{~B}$ and data not shown). The increase in the numbers of cells expressing intracellular TLR2 because of prior challenge, however, was more pronounced than the propagation of extracellular TLR2 ${ }^{+}$cells (Figure 5, $\mathrm{A}-\mathrm{C}$ ), and the signals detected were largely TLR2-specific (Figure 5, B and C).

Antibody-mediated interference with TLR2-dependent cell activation in vivo leading to cytokine and chemokine release into the serum. Next, we determined cytokine and chemokine serum concentrations in mice either pretreated or not pretreated with T2.5 and challenged with $\mathrm{P}_{3} \mathrm{CSK}_{4}$. While cytokine and chemokine concentrations were low in sera of untreated mice (see Methods), serum levels of TNF- $\alpha$, GRO $\alpha /$ KC (a murine homolog of human IL-8), IL-6, and IL-12p40 were significantly lower in mice preinjected with T2.5 than in controls upon challenge with $\mathrm{P}_{3} \mathrm{CSK}_{4}$ (Figure 6, A-D).

Antibody-mediated interference with systemic induction of shock-like syndromes through TLR2-specific challenge. Both a high-dose (microbial product only) and a low-dose model (additional sensitization with D-galactosamine) have been established for bacterial product-induced shock-like syndromes in mice (38). In order to interfere in a specific model of septic shock, we applied the bacterial lipopeptide analogue $\mathrm{P}_{3} \mathrm{CSK}_{4}$ (a TLR2 agonist) upon sensitization of mice with IFN- $\gamma$ and D-galactosamine $(39,40)$. Sensitization was used to mimic an underlying primary infection priming host defense. While mice that had received no mAb or conT2 30 minutes prior to injection succumbed to lethal toxemia within 24 hours, mice treated with T2.5 survived (Figure 7A). Intending to use a more complex challenge for a distinct shock model, we took advantage of the finding that shock-like syndrome induction by viable or heat-inactivated (h.i.) B. subtilis bacteria was TLR2- 

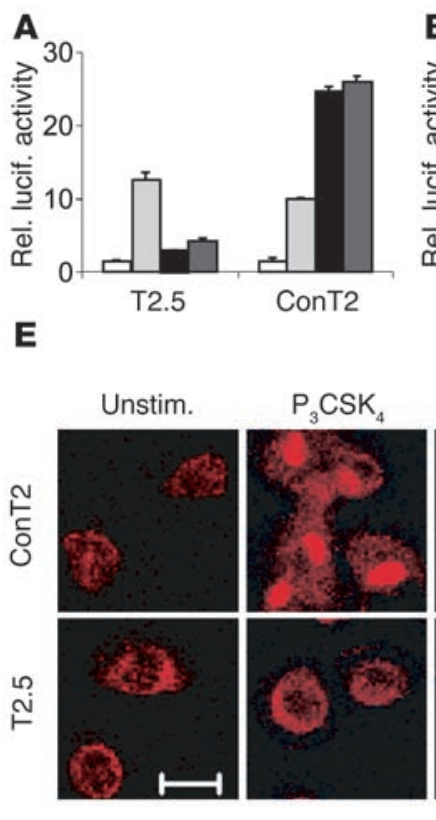
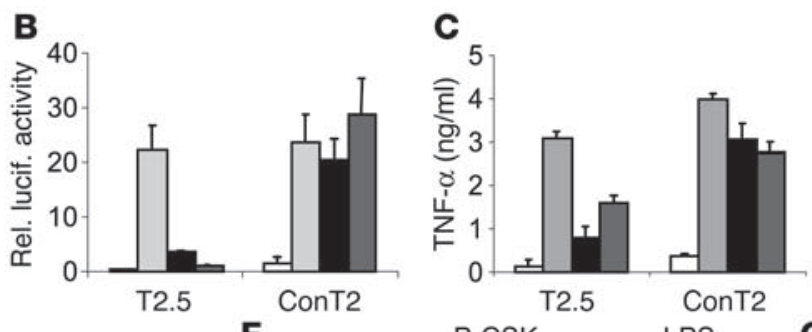

$\mathbf{F}$$$
\text { (1) }
$$

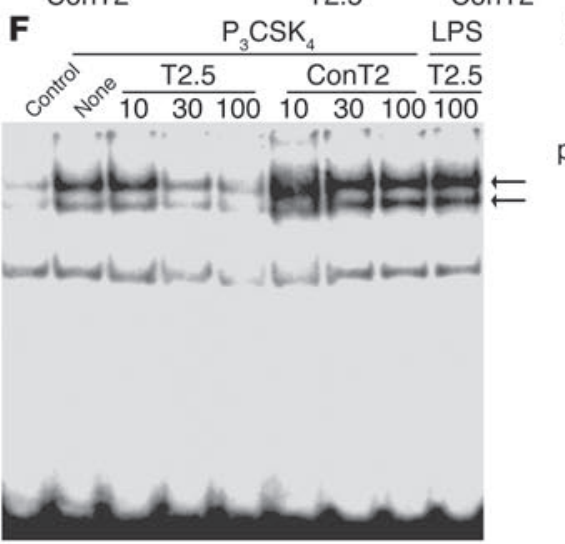

D

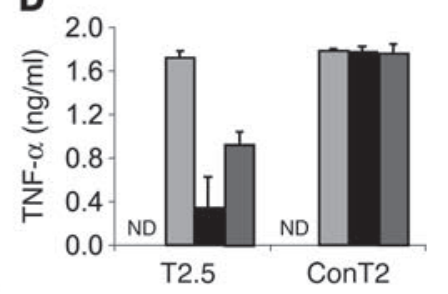

G

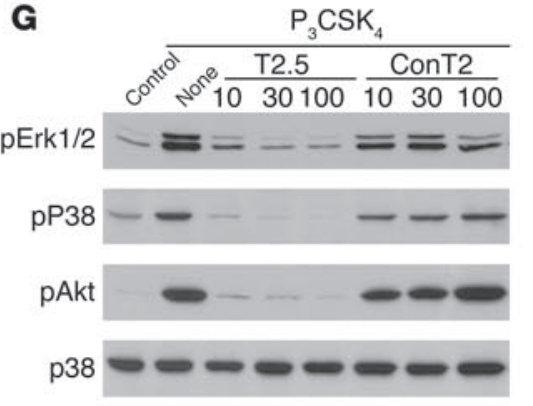

Figure 3

Inhibitory effect of mAb T2.5 on cell activation in vitro. (A-D) NF-кB-dependent luciferase activities in HEK293 cells overexpressing either murine (A) or human TLR2 (B), as well as TNF- $\alpha$ concentrations in supernatants of RAW264.7 (C) or primary murine macrophages (D) challenged with inflammatory agonists. Rel. lucif. activity, relative luciferase activity; ND, not detectable. Cells were incubated with T2.5 or conT2 only (white bars), or additionally challenged with IL-1 $\beta$ ( $\mathbf{A}$ and $\mathbf{B}$, light gray bars), ultrapure LPS (C and $\mathbf{D}$, medium gray bars), $\mathrm{P}_{3} \mathrm{CSK} \mathrm{K}_{4}$ (black bars), or h.i. B. subtilis (A-D, dark gray bars). (E) NF-KB/p65 nuclear translocation dependent on $m A b, P_{3} C S K_{4}$ challenge, or LPS challenge in human macrophages was analyzed by cytochemical staining. Unstim., unstimulated. Scale bar: $20 \mu \mathrm{m}$; magnification was equal for all recordings. ( $\mathbf{F}$ and $\mathbf{G}$ ) NF-KB-dependent EMSA was analyzed by application of nuclear extracts from RAW264.7 macrophages, and phosphorylation of MAPKs Erk1/2 (pErk1/2), p38 (pP38), and Akt (pAkt) was analyzed by application of total extracts from RAW264.7 macrophages. Cells were preincubated with the indicated amounts of $\mathrm{mAb}$ T2.5 or conT2 $(\mu \mathrm{g} / \mathrm{ml})$ and challenged with $\mathrm{P}_{3} \mathrm{CSK}_{4}$ or LPS subsequently for 90 minutes (F; arrows indicate specific NF-KB-DNA complexes) or 30 minutes (G; phosphorylation-independent p38-specific immunoblot analysis as positive control). Untreated cells were analyzed as controls (Control).

dependent. Clearance of B. subtilis, notably, was complete within 48 hours in TLR2 $2^{-/}$mice challenged intraperitoneally (i.p.) with viable $B$. subtilis at dosages lethal for wild-type mice. These findings encompassing TLR2 dependence in vivo indicated toxemia as the major pathophysiologic cause of wild-type mouse lethality in both experimental models $\left(\mathrm{P}_{3} \mathrm{CSK}_{4}\right.$-induced and B. subtilis-induced experimental shock-like syndrome; A. Grabiec and C.J. Kirschning, unpublished observations). Thus, mice were pretreated with different doses of T2.5 prior to administration of h.i. B. subtilis. While pretreatment with $1 \mathrm{mg}$ and $0.5 \mathrm{mg}$ of $\mathrm{T} 2.5$ protected mice from lethal toxemia (protective protocol), lower amounts were ineffective (Figure 7B). Next, aside from T2.5, conT2 was also applied prior to administration of a principally lethal dose of h.i. B. subtilis. In a separate group of mice, we first administered h.i. B. subtilis and applied T2.5 up to 4 hours later (therapeutic protocol). In the absence of sufficient dosage of T2.5, the high-dose h.i. B. subtilis challenge was lethal for all mice tested (Figure 7, B and C). However, when given T2.5 either 1 hour before or up to 2 hours after microbial challenge, all mice challenged with h.i. B. subtilis survived. Most notably, treatment with T2.5 even 3 hours after otherwise lethal injection saved $75 \%$ of mice challenged (Figure 7C). When the order of experimental T2.5 and h.i. B. subtilis application was reversed, a completely protective effect of $\mathrm{T} 2.5$ administration was evident if the bacterial challenge was started 3 hours later (Figure 7D). While T2.5 treatment was effective for two out of three mice even when applied for 4 hours, a protective effect was not detectable at the 5-hour and 6-hour time points in the respective experimental setting (Figure 7D).

\section{Discussion}

Our results suggest a therapeutically useful function of an antagonistic TLR2 $\mathrm{mAb}$ in TLR2-driven toxemia. We found that application of TLR2 agonists was lethal in two experimental models of septic shock and aimed to identify antibodies that recognize TLR2. One mAb, named T2.5, blocked mTLR2-dependent cell activation. T2.5 also blocked human TLR2 function, since subcellular NF- $\kappa \mathrm{B}$ translocation upon TLR2-specific challenge of primary human macrophages was inhibited upon its application. The neutralizing effect of T2.5 application is based on abrogation of TLR2ECD-agonist binding as revealed by SPR analysis upon immobilization of $\mathrm{P}_{3} \mathrm{CSK}_{4}$. Here we show that T2.5 prevents lethal shock-like syndromes induced by $\mathrm{P}_{3} \mathrm{CSK}_{4}$ or Gram-positive bacteria (B. subtilis) in mice.

The lack of TLR functions negatively affects humans, at least upon acute infection $(41,42)$. However, in a systemic model of polymicrobial sepsis encompassing standardized influx of the gut flora into the peritoneal cavity, mice benefit from the lack of TLR functions (43), which indicates TLR-dependent mediation of harmful effects in acute infection. Accordingly, blockage of LPS-binding protein (LBP) (44), as well as application of LBP, of peptides rep- 
A

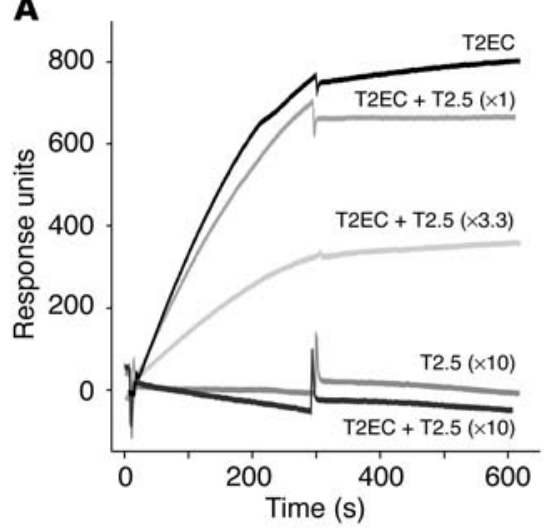

B

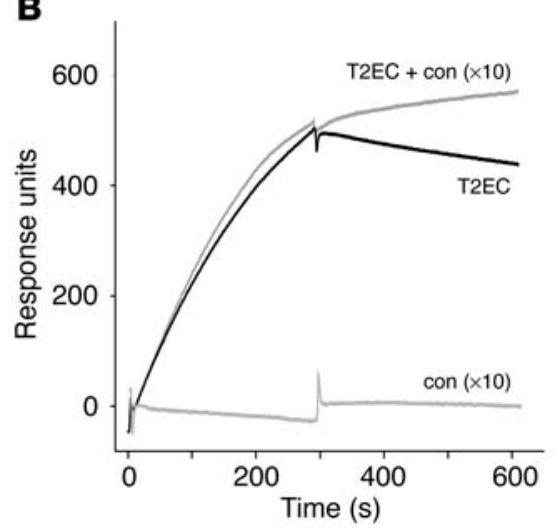

C

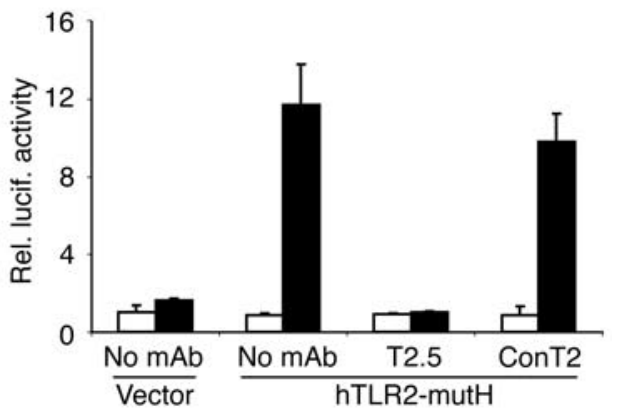

Figure 4

Molecular analysis of the effects of mAb T2.5 on TLR2ECD-P ${ }_{3} \mathrm{CSK}_{4}$ interaction. (A and B) Binding of recombinant TLR2ECD-Fc fusion protein (T2EC, positive controls) to immobilized $\mathrm{P}_{3} \mathrm{CSK}_{4}$ upon preincubation with T2.5 $(\mathrm{T} 2 \mathrm{EC}+\mathrm{T} 2.5)$ at different molar excesses $(\mathbf{A}, \times 1, \times 3.3, \times 10)$ or with an isotype-matched control mAb (T2EC + con) at tenfold molar excess only $(\mathbf{B}, \times 10)$. Binding was continuously monitored in an SPR biosensor device, and amounts of antibodies used to gain high molecular excess over T2EC (coincubation) were applied alone as negative controls (A, T2.5; B, Con). Response units at 300 seconds are a measure for $\mathrm{P}_{3} \mathrm{CSK}_{4}$-binding capacities of T2EC and T2EC plus mAb. (C) For analysis of approximate localization of T2.5 epitope within the TLR2ECD, a mutant human TLR2 construct lacking the N-terminal third of the LRR-rich ECD (hTLR2-mutH) was used for NF-kB-dependent luciferase assay upon transient transfection, preincubation with mAb (T2.5, conT2), and $\mathrm{P}_{3} \mathrm{CSK}_{4}$ challenge (black bars). Absence of $\mathrm{mAb}$ treatment (No mAb) and/or of $\mathrm{P}_{3} \mathrm{CSK}_{4}$ challenge (white bars), and empty vector (Vector), represent respective controls.

resenting its subdomains, or of bactericidal/permeability-increasing protein (BPI), has been effective in inhibiting LPS-induced pathology (45-49). Attempting to inhibit a TLR-specific immune activation as has been exemplified by systemic tolerance induction through TLR2-specific challenge prior to principally fatal microbial challenge (40), we applied an antagonistic mAb T2.5 raised against the murine TLR2ECD. Its application enabled analysis of murine and human TLR2 localization on the surface and inside of immune cells (Figures 1 and 2). Direct interaction between TLR2 and $\mathrm{P}_{3} \mathrm{CSK}_{4}$ was demonstrated and allowed comparison of the affinities of TLR2 and of the TLR2-T2.5 complex to this ligand. SPR analysis showed the direct and specific interaction between TLR2ECD and $\mathrm{P}_{3} \mathrm{CSK}_{4}$, as well as a specific and dose-dependent inhibition of this interaction by T2.5 (Figure 4, A and B), indicating that binding of T2.5 masked the ligand-binding domain in TLR2. Accordingly, T2.5 antagonized not specifically $\mathrm{P}_{3} \mathrm{CSK}_{4}$, but also h.i. B. subtilis, PGN, LTA, and TLR2-dependent cell activation induced by mycoplasmal macrophage-activating protein (Figure 3 and data not shown). Blockage was specific and dose-dependent (Figure 3). Taken together, these findings show that specific binding of ligands to a discrete site within the TLR2ECD is a prerequisite for TLR2-mediated signaling.

Surface expression of TLR2 in vivo was a precondition of systemic effects of T2.5 application. Relatively weak surface expression of TLR2 even upon LPS or bacterial challenge ex vivo (Figure 5 , A and B), however, was in contrast with relatively high surface expression on unchallenged primary murine (Figures $1 \mathrm{D}$ and 2B) as well as human myeloid cells upon in vitro culture (50). However, comparative TLR2 staining of nonpermeabilized and permeabilized cells indicated localization of a major portion of TLR2 in the intracellular compartment of murine CD11 $\mathrm{b}^{+}$and GR1 $1^{+}$cells, as well as human macrophages (Figure 5, Figure 2B, and data not shown). In fact, we noted increased surface and, to a larger extent, intracellular TLR2 expression in specific cell populations 24 hours after bacterial infection, which was similar upon LPS challenge (Figure 5 and data not shown). Weak unspecificity of intracellular staining with T2.5, detected mostly in permeabilized spleen cells, had to be taken into account (Figure 5C). The time course of TLR2 regulation in distinct immune cells upon microbial contact needs to be investigated in more detail, because it might determine the time frame within which intervention based on TLR2 blockage can be effective.

Perhaps it is the surprisingly low constitutive surface expression of TLR2 in host cells such as CD11b+ (macrophage) cells, $\mathrm{GR}^{+}$(granulocyte) cells, CD19+ ${ }^{+}$B) cells, and CD11 ${ }^{+}$(dendritic) cells in vivo (Figure 5 and data not shown) that explains the high efficacy of T2.5-mediated prevention of TLR2-driven hyperinflammation (Figures 6 and 7). Application of T2.5 30 minutes prior to application of a principally lethal dose of $\mathrm{P}_{3} \mathrm{CSK}_{4}$ or 1 hour prior to administration of a principally lethal dose of h.i. B. subtilis protected mice against the otherwise lethal effects of both stimulants (Figure 7, A-C), but not against the lethal effects of LPS (data not shown). In fact, B. subtilis-induced toxemia was prevented upon application of T2.5 2 hours or even 3 hours after shock-like syndrome induction $(100 \%$ or $75 \%$ of survival, respectively). In contrast, application of T2.5 was not effective after 4 hours (Figure 7C). However, the onset of septic shock upon acute infection in the clinical situation may be delayed as compared with sudden induction of toxemia by experimental injection of large amounts of stimulant and may allow interference within a larger time window. Our results indicate that complement-mediated depletion of TLR2 ${ }^{+}$cells is unlikely to be a mechanism of prevention of T2.5-dependent prevention of TLR2-driven shocklike syndrome, since application of the MTLR2-specific isotypematched $\mathrm{mAb}$ conT2 in vivo did not result in protection (Figure 7A). This is in line with reversibility of mAb-mediated TLR2 blockage within 5 hours (Figure 7D), which may be important for timely recovery of TLR2-dependent cellular responsiveness 
A
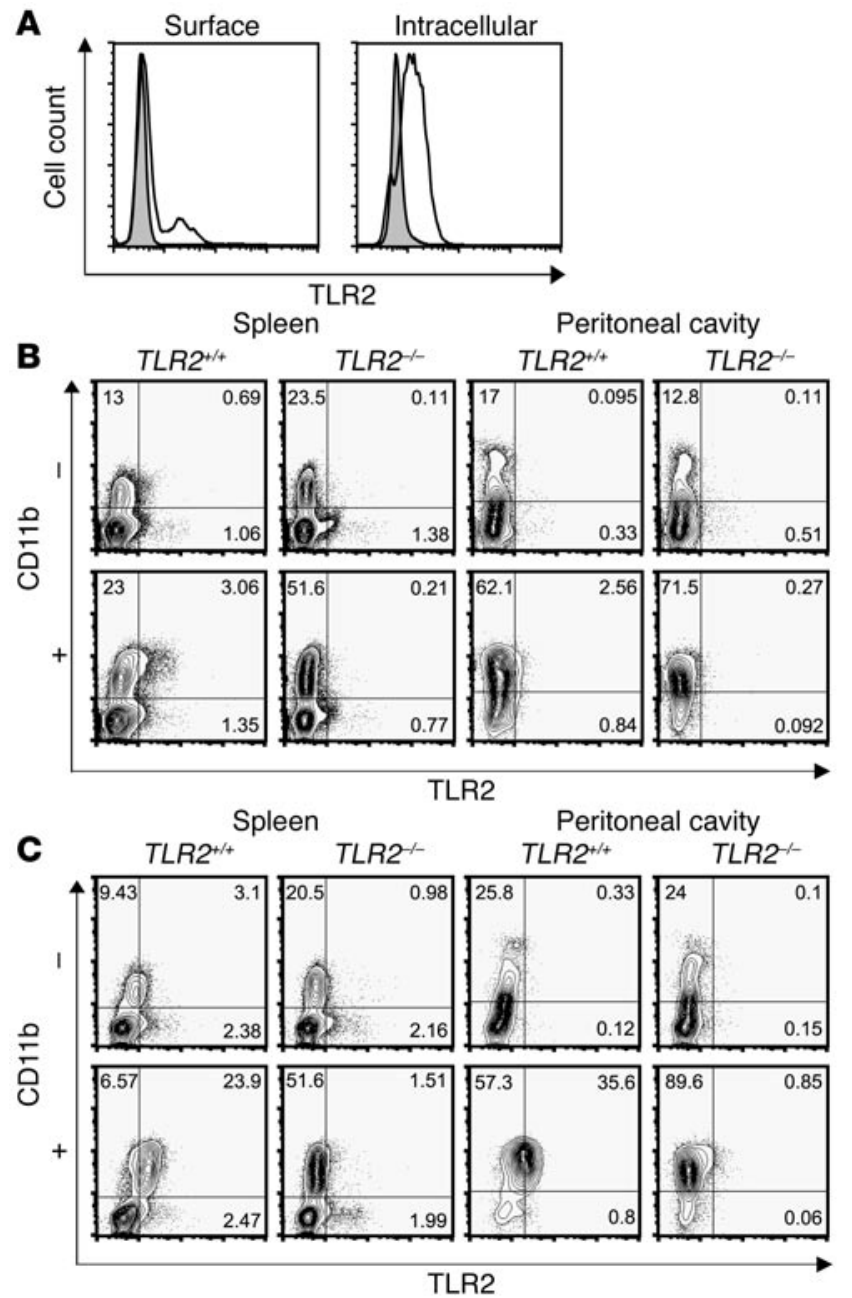

in later phases of sepsis at which diminished immune function is fatal (9). Systemic presence of T2.5 1 hour prior to challenge did not interfere with resistance of a TLR2 $2^{-/}$mouse challenged with h.i. B. subtilis at a dose that was lethal for wild-type mice in the absence of T2.5 application (data not shown). The demonstration of beneficial and specific effects of T2.5 in both a sensitizationdependent and a high-dose TLR2-specific experimental model may support transferability of our results to elimination of the TLR2-dependent share in septic shock induction (9). Specifically, TLR2 blockage upon antibiotic therapy may substantially contribute to prevention of an excessive host immune reaction upon sudden release of large amounts of microbial products from disintegrating microbial cells. It may have to be complemented by blockage of further surface receptors, for which TLR4 is a prime

\section{Figure 6}

Inhibitory effect of mAb T2.5 on host activation by microbial challenge in vivo. Mice were pretreated i.p. with $1 \mathrm{mg}$ mAb T2.5 (black bars) or left untreated (white bars). Mice were challenged i.p. with $\mathrm{P}_{3} \mathrm{CSK}_{4}$ and D-galactosamine after 1 hour and sacrificed 2 or 4 hours later $(n=4$ for each group at each time point). Serum concentrations of TNF- $\alpha(\mathbf{A})$, GRO $\alpha /$ KC (human IL-8 homolog) (B), IL-6 (C), and IL-12p40 (D) were analyzed by ELISA. ${ }^{*} P<0.05,{ }^{* *} P<0.005,{ }^{* *} P<0.001$, Student's $t$ test for unconnected samples.

\section{Figure 5}

TLR2 expression ex vivo immediately after primary cell isolation. Flow cytometry of splenocytes and peritoneal washout cells from wildtype $\left(T L R 2^{+/+}\right)$and $T L R 2^{-/-}$mice ex vivo immediately after isolation ( $n=5$, cells pooled for each sample). (A) CD11b+ splenocytes from mice challenged with LPS for 24 hours were analyzed for surface and intracellular TLR2 expression by staining with T2.5 (bold line, $T L R 2^{+/+}$; filled area, $T L R 2^{--}$). (B and C) For analysis of TLR2 regulation upon infection, mice were either left uninfected $(-)$ or infected with $B$. subtilis and sacrificed after 24 hours (+). Upon staining of CD11b, cells were stained with T2.5 (TLR2) either without permeabilization (B) or after permeabilization (C). Numbers in quadrants represent the percentage of single- or double-stained cells with respect to the total number of viable cells analyzed.

candidate, in order to facilitate inhibition of cell activation. Conversely, failure of therapy to compensate for a decrease in biocidal immune cell activity upon TLR blockage by antibiotic treatment might compromise a beneficial outcome (13).

We have identified exclusively antagonistic or neutral TLR2specific $\mathrm{mAb}$ 's, and antagonistic properties have recently been demonstrated in vitro also for two different human TLR2-specific mAb's $(28,51)$. Active complex formation of TLRs as compared with receptors for which agonistic antibodies have been identified might differ. However, T2.5 antagonized TLR2 function through inhibition of ligand-TLR2-complex formation (Figure $4 \mathrm{~A}$ ), which is a prerequisite of TLR2-driven cell activation. T2.5 may therefore recognize the possibly single ligand-binding site within the C-terminal portion of the TLR2ECD. We expect that identification of the epitope will show its conservation between mice and humans. In conclusion, our results implicate antibodymediated TLR blockage on immune cells as a promising strategy for attenuation of potentially fatal host-response amplification in the course of acute infection.

\section{Methods}

Material. Overnight B. subtilis (DSMZ.1087) cultures in brain-heart medium containing approximately $1 \times 10^{9} \mathrm{CFUs} / \mathrm{ml}$ were used immediately or heat-inactivated at $56^{\circ} \mathrm{C}$ for 50 minutes. Synthetic $\mathrm{P}_{3} \mathrm{CSK}_{4}$ and, as a negative control, $\mathrm{PHCSK}_{4}$, a nonstimulatory derivative thereof (52), were purchased from EMC microcol-
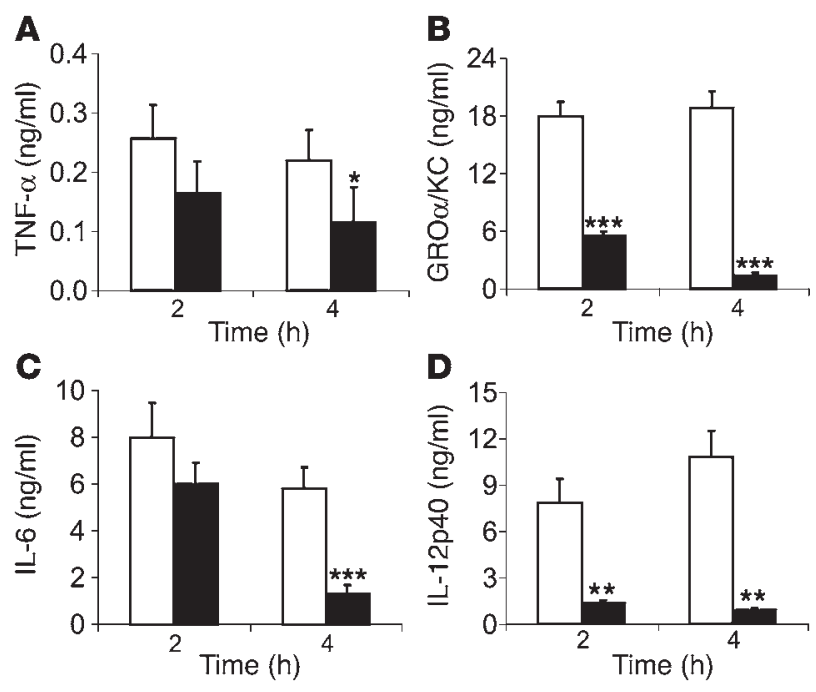
A
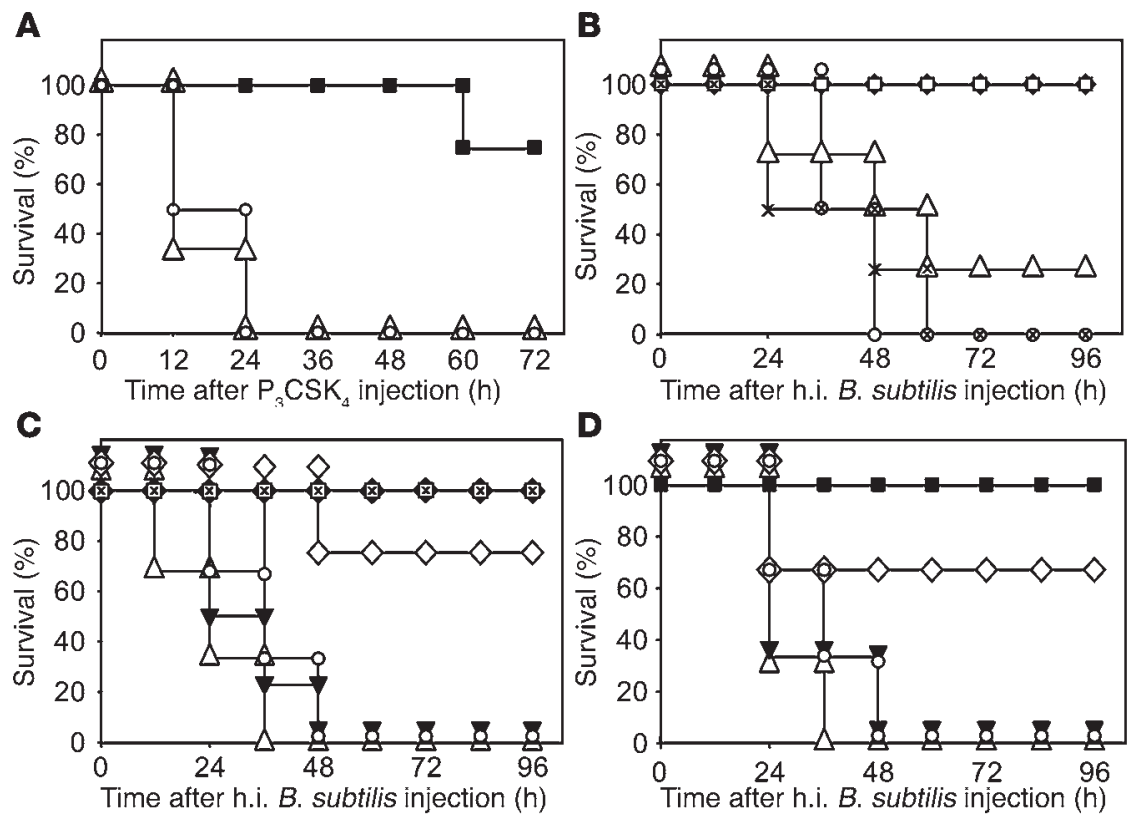

D

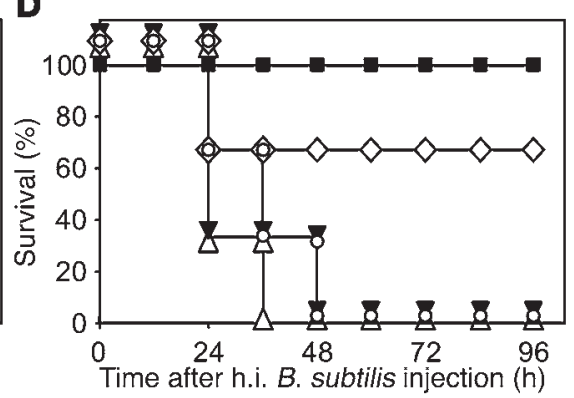

\section{Figure 7}

Effects of mAb T2.5 administration on viability after TLR2-specific systemic challenge. (A) IFN- $\gamma$ - and D-galactosamine-sensitized mice received no mAb, $1 \mathrm{mg}$ of $\mathrm{mAb}$ T2.5, or $1 \mathrm{mg}$ of conT2 i.p. 30 minutes prior to microbial challenge with bacterial lipopeptide analogue $\mathrm{P}_{3} \mathrm{CSK}_{4}$ (open circles, no mAb, $n=4$; open triangles, mAb conT2, $n=3$; filled squares, mAb T2.5, $n=4$ ). (B-D) Mice challenged with a high dose of h.i. B. subtilis were left untreated, treated 1 hour later with the indicated dosages of mAb T2.5 (B; filled diamonds, $1 \mathrm{mg}, n=3$; open squares, $0.5 \mathrm{mg}, n=3$; open triangles, $0.25 \mathrm{mg}, n=4$; х's, $0.13 \mathrm{mg}, n=4$; open circles, no mAb T2.5, $n=4$ ), or treated with $1 \mathrm{mg}$ of mAb's at the different time points indicated below (C and D). (C) TLR2-specific $\mathrm{mAb}$ was administered before $(-)$ or after $(+)$ bacterial challenge (filled inverted triangles, no mAb, $n=8$; open circles, mAb conT2, -1 hour, $n=3$; filled diamonds, mAb T2.5, -1 hour, $n=4$; open squares, mAb T2.5, +1 hour, $n=3$; x's, mAb T2.5, +2 hours, $n=3$; open diamonds, mAb T2.5, +3 hours, $n=4$; open triangles, mAb T2.5, +4 hours, $n=3$ ). (D) TLR2specific mAb T2.5 was administered before (-) bacterial challenge (open triangles, no mAb; filled squares, mAb T2.5, -3 hours; open diamonds, mAb T2.5, -4 hours; open circles, mAb T2.5, -5 hours; filled inverted triangles, mAb T2.5, -6 hours; $n=3$ for all groups).

lections $\mathrm{GmbH}$ (Tuebingen, Germany); both carried biotin tags. Ultrapure LPS from Salmonella minnesota Re595 was from List Laboratory (Campbell, California, USA), recombinant murine IFN- $\gamma$ and IL- $1 \beta$ were from PeproTech EC Ltd. (London, United Kingdom), and D-galactosamine was from Sigma-Aldrich Chemie $\mathrm{GmbH}$ (Deisenhofen, Germany).

Mice. Matched groups of wild-type (TLR2 $\left.{ }^{+/+}\right) \mathrm{C} 57 \mathrm{BL} / 6$ and TLR2/- (39) mice generated by Deltagen Inc. (Redmond City, California, USA) were kindly provided by Tularik Inc. (South San Francisco, California, USA) and crossed ninefold from a mixed Sv129×C57BL/6 toward a C57BL/ 6 genetic background. Experiments were approved by the government of Upper Bavaria, Germany.

Generation of TLR2ECD-specific antibodies and ELISA. A cDNA fragment encoding the N-terminal 587 amino acids of mTLR2 (53) was amplified from an RAW264.7 cell cDNA library (Advantage kit; BD Biosciences Clontech, Heidelberg, Germany). The murine TLR2ECD was fused to a C-terminal thrombin cleavage site followed by a human IgGFC $\gamma$ moiety (T2EC). The murine TLR2ECD protein was purified upon overexpression in HEK293 cells and thrombin digestion. A TLR2 $2^{-/-}$mouse was immunized three times within 8 weeks by i.p. injection of $50 \mu \mathrm{g}$ of TLR2ECD and $10 \mathrm{nmol}$ of a thioated DNA oligonucleotide (5'-TCCATGACGTTCCTGA-
3'; TIB MOLBIOL, Berlin, Germany). Its splenocytes were fused with murine P3X cells, and hybridomas were selected (54). Monoclonal antibody specificities for TLR2ECD, as well as cytokine and chemokine concentrations, in cell supernatants or murine sera were analyzed by ELISA (R\&D Systems Inc., Minneapolis, Minnesota, USA). Significance of serum-concentration differences was determined by application of the Student's $t$ test for unconnected samples.

Flow cytometry. Stably transfected HEK293 cell clones, as well as uninduced peritoneal washout macrophages, were cultured overnight as described previously (37). Flow cytometry was performed upon staining with $\mathrm{T} 2.5$ and a secondary mouse IgG-specific $\mathrm{mAb}$, as well as affinity-purified polyclonal antisera specific for the murine TLR2ECD (55) or the Flag-tag (Sigma-Aldrich Chemie $\mathrm{GmbH}$ ) and a rabbit IgG-specific secondary $\mathrm{mAb}$. Secondary mAb's were phycoerythrinlabeled (BD Biosciences Pharmingen, Heidelberg, Germany). For establishment of MTLR2 expression analysis in primary cells, surface and intracellular T2.5-dependent staining of CD $11 b^{+}$splenocytes (54) from wild-type versus TLR2 $2^{-/-}$mice challenged with LPS (0.5 mg, i.p., 24 hours) was compared by flow cytometry (CyAn; DakoCytomation, Fort Collins, Colorado, USA). Cells were stained with photoactivated ethidium monoazide (Molecular Probes Europe BV, Amsterdam, The Netherlands) immediately upon isolation, followed by TLR2-specific surface staining, or intracellular staining (Cytofix/ Cytoperm; BD Pharmingen). In order to analyze TLR2 expression in uninfected or $B$. subtilis-infected mice $\left(5 \times 10^{8}\right.$ CFUs, i.p., 24 hours $)$, peritoneal washout cells and splenocytes (54) from five uninfected or infected wildtype or TLR2 $2^{-1-}$ mice were pooled. Fluorescence-labeled cell surface marker antibodies (BD Pharmingen) and T2.5 counterstained with secondary anti-mIgG1 were used as indicated.

Immunoprecipitation and immunoblot analysis. Lysates of FlagTLR2-transfected HEK293 cells or macrophages were mixed with $1 \mu \mathrm{g}$ of antibody and protein $\mathrm{G}$ beads (Santa Cruz Biotechnology Inc., Santa Cruz, California, USA) for overnight precipitation. Immune complexes or cell lysates were analyzed by immunoblot analysis as described previously (37). Precipitations were controlled by application of Flag-specific (mAb M2; Sigma-Aldrich Chemie $\mathrm{GmbH}$ ) or protein $\mathrm{G}$ beads only. Flag- or mTLR2-specific antisera were applied for immunoblot analyses of HEK293 or RAW264.7 cell lysates, respectively. In contrast, total lysates of macrophages were analyzed for phosphorylation of kinases as indicated.

Cytochemical staining of TLR2 or NF- $\mathrm{K} B$. Transfected HEK293 cell clones, as well as primary murine or human macrophages, the latter isolated as $\mathrm{CD} 14^{+}$peripheral blood leukocytes and cultured in $20 \%$ of autologous serum (56), were grown on slides. Cells were washed with PBS, permeabilized, and incubated with $5 \mu \mathrm{g} / \mathrm{ml}$ TLR2-specific mAb or anti-NF- $\mathrm{KB} / \mathrm{p} 65$ (polyclonal 
rabbit; Santa Cruz Biotechnology Inc.) (37). Specific secondary anti-IgG antibodies labeled with Alexa Fluor 546 (anti-TLR2) or Cy5 (anti-NF-кB; both from BD Biosciences Pharmingen) were applied. Cell membranes were stained with labeled concanavalin A (Molecular Probes Europe BV).

Inbibition of TLR2-dependent cell activation in vitro and in vivo. Transiently transfected HEK293 cells as well as murine RAW264.7 and primary macrophages were used. Fifty micrograms per milliliter of antibodies were applied 30 minutes prior to challenge with 100 $\mathrm{ng} / \mathrm{ml}$ of LPS, IL- $1 \beta$, or $\mathrm{P}_{3} \mathrm{CSK}_{4}$ or $1 \times 10^{6} \mathrm{CFUs} / \mathrm{ml}$ of h.i. B. subtilis. HEK293 cells were cotransfected with reporter (57), human wildtype TLR2, human mutant TLR2 (lacking the N-terminal third of the LRR-rich domain; ref. 37), or mTLR2, as well as MD2 and CD14 (provided by Tularik Inc., South San Francisco, California, USA; D. Golenbock, University of Massachusetts Medical School, Worcester, Massachusetts, USA; H. Heine, Research Center Borstel, Borstel, Germany; and K. Miyake, University of Tokyo, Tokyo, Japan) expression plasmids, and NF- $\mathrm{KB}-$ dependent reporter gene activity was assayed after 6 hours of stimulation. TNF- $\alpha$ concentrations in supernatants of RAW264.7 and primary murine macrophages were analyzed 24 hours after challenge, and NF-кB translocation in human macrophages (56) was analyzed 90 minutes after challenge. RAW264.7 macrophages were used for analysis of challenge and of

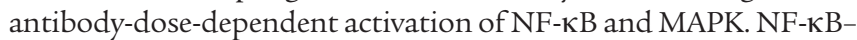
specific EMSA and p38, Erk1/2, and Akt phosphorylation-specific immunoblot analysis (Cell Signaling Technology, Frankfurt, Germany) were carried out. Prior to TLR-specific challenge of $1 \times 10^{6}$ cells, as described above, for 90 minutes (EMSA) or 30 minutes (kinase-phosphorylation analysis) (37), antibody was administered at various concentrations. For analysis of TLR2 inhibition in vivo, mice were injected i.p. with $1 \mathrm{mg}$ of T2.5 or left untreated. One hour later, $100 \mu \mathrm{g}$ of $\mathrm{P}_{3} \mathrm{CSK}_{4}$ and $20 \mathrm{mg}$ of D-galactosamine were injected i.p. Serum concentrations of TNF- $\alpha$, GRO $\alpha / \mathrm{KC}$ (murine homolog of human IL-8), IL-6, and IL-12p40 in five unchallenged control mice were $0.05 \mathrm{ng} / \mathrm{ml}, 0.43 \mathrm{ng} / \mathrm{ml}$, not detectable, and 0.44 $\mathrm{ng} / \mathrm{ml}$, respectively. Significance of results was determined by the Student's $t$ test for unconnected samples.

$S P R$ biosensor measurements. Real-time binding analysis was performed using SPR detection on a Biacore X device (Biacore AB, Uppsala, Sweden). The two flow cells (FCs) of a streptavidin-precoated chip were loaded with biotinylated $\mathrm{PHCSK}_{4}$ (FC1) and $\mathrm{P}_{3} \mathrm{CSK}_{4}$ (FC2), respectively. Specific binding of a recombinant T2EC protein was controlled by application of a human $\mathrm{mAb}$ carrying the same Fcy domain. This antibody did not bind in either FC1 or FC2 (data not shown). After prior incubation in $45 \mu \mathrm{l}$ of running buffer (50 $\mathrm{mM}$ morpholino ethane sulfonic acid, 150 $\left.\mathrm{mM} \mathrm{MgCl}_{2}, \mathrm{pH} 6.5\right)$ at $25^{\circ} \mathrm{C}$ for 15 minutes, $200 \mathrm{nmol}$ of purified T2EC alone (maximum control) or in combination with mAb's
(T2.5 or an isotype-matched irrelevant mAb at the molar excesses indicated) was injected over FC1 and FC2 at a flow rate of $10 \mu \mathrm{l} /$ min. As negative control, mAb's alone were administered at the highest amounts also used for blocking analysis of TLR2 ligand binding. The values obtained upon continuous resonance monitoring at $25^{\circ} \mathrm{C}$ over 570 seconds (delay time 300 seconds) from the control FC1 were subtracted from the respective values resulting from simultaneously performed analysis of FC2. Generally, biomolecular interaction between receptor and its respective ligands immobilized on the sensor chip is optically monitored as a function of time and expressed in response units. Regeneration of the chip was achieved by washes with $50 \mathrm{mM} \mathrm{NaOH}$ and $1 \mathrm{M} \mathrm{NaCl}$ and extensive re-equilibration with running buffer.

Systemic induction of shock-like syndrome. In an experimental sensitization-dependent model (39), mice were injected i.v. with 1.25 $\mu \mathrm{g}$ of murine IFN- $\gamma$. Twenty minutes later, mice were injected i.p. with doses of $\mathrm{mAb}$ as indicated. Fifty minutes after IFN- $\gamma$ injection, $100 \mu \mathrm{g}$ of synthetic $\mathrm{P}_{3} \mathrm{CSK}_{4}$ and $20 \mathrm{mg}$ of D-galactosamine were injected i.p. as well. The experimental high-dose shock model encompassed a single i.p. injection of $1 \times 10^{10} \mathrm{CFUs}$ of h.i. B. subtilis, with i.p. injection of $1 \mathrm{mg}$ of $\mathrm{mAb} 1$ hour to 6 hours earlier or 1 hour to 4 hours later as indicated. Survival was monitored and did not change within 7 days after injection after the latest time points indicated in Figure 7.

\section{Acknowledgments}

We thank S. Fichte and M. Meyer for excellent technical assistance, S. Linder and B. Boehlig for provision of human primary macrophages, and K.H. Wiesmuller and R. Spohn for their generous support. We are also thankful to G. Hacker, H. Hochrein, and T. Miethke for helpful discussions, as well as I. Forster and M. Lochner for help with hybridoma culture and antibody purification. G. Meng was supported by Sonderforschungsbereich grant SFB576/A1. A. Grabiec and S. Bauer were supported by Deutsche Forschungsgemeinschaft grants Ki 591/1-1 and Ba 1618/3-1, respectively. D.H. Busch and M. Schiemann are members of the GSF Clinical Cooperation Group "Vaccinology."

Received for publication December 9, 2003, and accepted in revised form March 23, 2004.

Address correspondence to: Carsten J. Kirschning, Institute of Medical Microbiology, Immunology, and Hygiene, Technical University of Munich, Trogerstrasse 24, 81675 Munich, Germany. Phone: 49-89-4140-4132; Fax: 49-89-4140-4139; E-mail: carsten. kirschning@lrz.tum.de.

Guangxun Meng and Mark Rutz contributed equally to this work.
1. Medzhitov, R., and Janeway, C.A. 1997. Innate immunity: the virtues of a nonclonal system of recognition. Cell. 91:295-298.

2. Takeda, K., Kaisho, T., and Akira, S. 2003. Toll-like receptors. Annu. Rev. Immunol. 21:335-376.

3. Raetz, C.R., et al. 1991. Gram-negative endotoxin: an extraordinary lipid with profound effects on eukaryotic signal transduction. FASEB J. 5:2652-2660.

4. Ulevitch, R.J., and Tobias, P.S. 1995. Receptordependent mechanisms of cell stimulation by bacterial endotoxin. Annu. Rev. Immunol. 13:437-457.

5. Lien, E., et al. 2000. Toll-like receptor 4 imparts ligand-specific recognition of bacterial lipopolysaccharide. J. Clin. Invest. 105:497-504.
6. Beutler, B., and Rietschel, E.T. 2003. Timeline. Innate immune sensing and its roots: the story of endotoxin. Nat. Rev. Immunol. 3:169-176.

7. Wagner, H. 2001. Toll meets bacterial CPG-DNA. Immunity. 14:499-502.

8. Cohen, J. 2002. The immunopathogenesis of sepsis. Nature. 420:885-891.

9. Hotchkiss, R.S., and Karl, I.E. 2003. The pathophysiology and treatment of sepsis. N. Engl. J. Med. 348:138-150.

10. Martin, G.S., Mannino, D.M., Eaton, S., and Moss, M. 2003. The epidemiology of sepsis in the United States from 1979 through 2000. N. Engl. J. Med. 348:1546-1554.

11. Roger, T., David, J., Glauser, M.P., and Calandra,
T. 2001. MIF regulates innate immune responses through modulation of Toll-like receptor 4. Nature. 414:920-924.

12. Schimke, J., Mathison, J., Morgiewicz, J., and Ulevitch, R.J. 1998. Anti-CD14 mAb treatment provides therapeutic benefit after in vivo exposure to endotoxin. Proc. Natl. Acad. Sci. U. S. A. 95:13875-13880.

13. Bochud, P.Y., and Calandra, T. 2003. Pathogenesis of sepsis: new concepts and implications for future treatment. BMJ. 326:262-266.

14. Wang, H., et al. 1999. HMG-1 as a late mediator of endotoxin lethality in mice. Science. 285:248-251.

15. Jin, H., et al. 1994. Protection against rat endotoxic shock by p55 tumor necrosis factor (TNF) recep- 
tor immunoadhesin: comparison with anti-TNF monoclonal antibody. J. Infect. Dis. 170:1323-1326.

16. Yoon, D.Y., and Dinarello, C.A. 1998. Antibodies to domains II and III of the IL-1 receptor accessory protein inhibit IL-1 beta activity but not binding: regulation of IL-1 responses is via type I receptor, not the accessory protein. J. Immunol. 160:3170-3179.

17. Ito, H. 2003. Anti-interleukin-6 therapy for Crohn's disease. Curr. Pharm. Des. 9:295-305.

18. Reimold, A.M. 2003. New indications for treatment of chronic inflammation by TNF-alpha blockade. Am. J. Med. Sci. 325:75-92.

19. Dziarski, R., Jin, Y.P., and Gupta, D. 1996. Differential activation of extracellular signal-regulated kinase (ERK) 1, ERK2, p38, and c-Jun NH2-terminal kinase mitogen-activated protein kinases by bacterial peptidoglycan. J. Infect. Dis. 174:777-785.

20. Kengatharan, K.M., De Kimpe, S., Robson, C., Foster, S.J., and Thiemermann, C. 1998. Mechanism of gram-positive shock: identification of peptidoglycan and lipoteichoic acid moieties essential in the induction of nitric oxide synthase, shock, and multiple organ failure. J. Exp. Med. 188:305-315.

21. Schleifer, K.H., and Kandler, O. 1972. Peptidoglycan types of bacterial cell walls and their taxonomic implications. Bacteriol. Rev. 36:407-477.

22. Fischer, W. 2000. Phosphocholine of pneumococcal teichoic acids: role in bacterial physiology and pneumococcal infection. Res. Microbiol. 151:421-427.

23. Fan, X., et al. 1999. Structures in Bacillus subtilis are recognized by CD14 in a lipopolysaccharide binding protein-dependent reaction. Infect. Immun. 67:2964-2968.

24. Bessler, W.G., Johnson, R.B., Wiesmuller, K., and Jung, G. 1982. B-lymphocyte mitogenicity in vitro of a synthetic lipopeptide fragment derived from bacterial lipoprotein. Hoppe Seylers Z. Physiol. Chem. 363:767-770.

25. Wiedmann, M., Arvik, T.J., Hurley, R.J., and Boor, K.J. 1998. General stress transcription factor sig$\mathrm{maB}$ and its role in acid tolerance and virulence of Listeria monocytogenes. J. Bacteriol. 180:3650-3656.

26. Schwandner, R., Dziarski, R., Wesche, H., Rothe, M., and Kirschning, C.J. 1999. Peptidoglycan- and lipoteichoic acid-induced cell activation is mediated by toll-like receptor 2. J. Biol. Chem. 274:17406-17409.

27. Yoshimura, A., et al. 1999. Cutting edge: recognition of gram-positive bacterial cell wall components by the innate immune system occurs via tolllike receptor 2. J. Immunol. 163:1-5.

28. Flo, T.H., et al. 2000. Human toll-like receptor 2 mediates monocyte activation by Listeria monocytogenes, but not by group B streptococci or lipopolysaccharide. J. Immunol. 164:2064-2069.

29. Takeuchi, O., Hoshino, K., and Akira, S. 2000. Cutting edge: TLR2-deficient and MyD88-deficient mice are highly susceptible to Staphylococcus aureus infection. J. Immunol. 165:5392-5396.

30. Edelson, B.T., and Unanue, E.R. 2002. MyD88dependent but Toll-like receptor 2 -independent innate immunity to Listeria: no role for either in macrophage listericidal activity. J. Immunol. 169:3869-3875.

31. Lembo, A., et al. 2003. Differential contribution of Toll-like receptors 4 and 2 to the cytokine response to Salmonella enterica serovar Typhimurium and Staphylococcus aureus in mice. Infect. Immun. 71:6058-6062.

32. Ozinsky, A., et al. 2000. The repertoire for pattern recognition of pathogens by the innate immune system is defined by cooperation between toll-like receptors. Proc. Natl. Acad. Sci. U. S. A. 97:13766-13771.

33. Takeuchi, O., et al. 2001. Discrimination of bacterial lipoproteins by Toll-like receptor 6. Int. Immunol. 13:933-940.

34. Alexopoulou, L., et al. 2002. Hyporesponsiveness to vaccination with Borrelia burgdorferi OspA in humans and in TLR1- and TLR2-deficient mice. Nat. Med. 8:878-884.

35. Mitsuzawa, H., et al. 2001. Extracellular Tolllike receptor 2 region containing Ser40-Ile64 but not Cys30-Ser39 is critical for the recognition of Staphylococcus aureus peptidoglycan. J. Biol. Chem. 276:41350-41356.

36. Rock, F.L., Hardiman, G., Timans, J.C., Kastelein, R.A., and Bazan, J.F. 1998. A family of human receptors structurally related to Drosophila Toll. Proc. Natl. Acad. Sci. U. S. A. 95:588-593.

37. Meng, G., et al. 2003. Cellular recognition of tri/di-palmitoylated peptides is independent from a domain encompassing the $\mathrm{N}$-terminal seven leucine rich repeat (LRR) / LRR-like motifs of TLR2. J. Biol. Chem. 278:39822-39829.

38. Freudenberg, M.A., and Galanos, C. 1991. Tumor necrosis factor alpha mediates lethal activity of killed gram-negative and gram-positive bacteria in D-galactosamine-treated mice. Infect. Immun. 59:2110-2115

39. Werts, C., et al. 2001. Leptospiral lipopolysaccharide activates cells through a TLR2-dependent mechanism. Nat. Immunol. 2:346-352.

40. Wang, J.H., et al. 2003. Cutting edge: bacterial lipoprotein induces endotoxin-independent tolerance to septic shock. J. Immunol. 170:14-18.

41. Picard, C., et al. 2003. Pyogenic bacterial infections in humans with IRAK-4 deficiency. Science. 299:2076-2079.

42. Medvedev, A.E., et al. 2003. Distinct mutations in IRAK-4 confer hyporesponsiveness to lipopolysaccharide and interleukin- 1 in a patient with recurrent bacterial infections. J. Exp. Med. 198:521-531.

43. Weighardt, H., et al. 2002. Cutting edge: myeloid differentiation factor 88 deficiency improves resistance against sepsis caused by polymicrobial infec- tion. J. Immunol. 169:2823-2827.

44. Gallay, P., Heumann, D., Le Roy, D., Barras, C., and Glauser, M.P. 1994. Mode of action of antilipopolysaccharide-binding protein antibodies for prevention of endotoxemic shock in mice. Proc. Natl. Acad. Sci. U. S. A. 91:7922-7926.

45. Lamping, N., et al. 1998. LPS-binding protein protects mice from septic shock caused by LPS or gramnegative bacteria. J. Clin. Invest. 101:2065-2071.

46. Dankesreiter, S., Hoess, A., Schneider-Mergener, J., Wagner, H., and Miethke, T. 2000. Synthetic endotoxin-binding peptides block endotoxin-triggered TNF-alpha production by macrophages in vitro and in vivo and prevent endotoxin-mediated toxic shock. J. Immunol. 164:4804-4811.

47. Gray, P.W., et al. 1989. Cloning of the cDNA of a human neutrophil bactericidal protein. Structural and functional correlations. J. Biol. Chem. 264:9505-9509.

48. Jin, H., et al. 1995. Protection against endotoxic shock by bactericidal/permeability-increasing protein in rats. J. Clin. Invest. 95:1947-1952.

49. Levin, M., et al. 2000. Recombinant bactericidal/ permeability-increasing protein (rBPI21) as adjunctive treatment for children with severe meningococcal sepsis: a randomised trial. rBPI21 Meningococcal Sepsis Study Group. Lancet. 356:961-967.

50. Krutzik, S.R., et al. 2003. Activation and regulation of Toll-like receptors 2 and 1 in human leprosy. Nat. Med. 9:525-532.

51. Brightbill, H.D., et al. 1999. Host defense mechanisms triggered by microbial lipoproteins through toll-like receptors. Science. 285:732-736.

52. Wiesmuller, K.H., Jung, G., and Hess, G. 1989. Novel low-molecular-weight synthetic vaccine against foot-and-mouth disease containing a potent B-cell and macrophage activator. Vaccine. 7:29-33.

53. Heine, H., et al. 1999. Cutting edge: cells that carry a null allele for toll-like receptor 2 are capable of responding to endotoxin. J. Immunol. 162:6971-6975.

54. Coligan, J.E., Kruisbeek, A.M., Margulies, D.H., Shevach, E.M., and Strobe, W. 1990. Current protocols in immunology. John Wiley \& Sons Inc. New York, New York, USA. 2.5.1-2.7.12.

55. Sing, A., et al. 2002. Yersinia V-antigen exploits toll-like receptor 2 and CD14 for interleukin 10-mediated immunosuppression. J. Exp. Med. 196:1017-1024.

56. Linder, S., Nelson, D., Weiss, M., and Aepfelbacher, M. 1999. Wiskott-Aldrich syndrome protein regulates podosomes in primary human macrophages. Proc. Natl. Acad. Sci. U. S. A. 96:9648-9653.

57. Schindler, U., and Baichwal, V.R. 1994. Three NFkappa $\mathrm{B}$ binding sites in the human E-selectin gene required for maximal tumor necrosis factor alphainduced expression. Mol. Cell. Biol. 14:5820-5831. 